11

\title{
Структура и оптические характеристики пленок ниобатов бария-стронция на подложках $\mathrm{Al}_{2} \mathrm{O}_{3}$
}

\author{
(C) А.В. Павленко ${ }^{1,2}$, С.В. Кара-Мурза ${ }^{3}$, А.П. Корчикова ${ }^{3}$, А.А. Тихий ${ }^{3}$, \\ Д.В. Стрюков ${ }^{1}$, Н.В. Ковтун ${ }^{1}$ \\ ${ }^{1}$ Южный научный центр РАН, \\ 344006 Ростов-на-Дону, Россия \\ ${ }^{2}$ Научно-исследовательский институт физики ЮФУ, \\ 344090 Ростов-на-Дону, Россия \\ 3 Луганский национальный университет им. Тараса Шевченко, \\ 91011 Луганск, Украина \\ e-mail: Antvpr@mail.ru
}

Поступила в редакцию 15.06.2018 г.

В окончательной редакции 26.10.2018 г.

Принята к публикации 04.12.2018 г.

\begin{abstract}
Проведены исследования структуры и оптических характеристик тонких пленок сегнетоэлектрикарелаксора $\mathrm{Ba}_{0.5} \mathrm{Sr}_{0.5} \mathrm{Nb}_{2} \mathrm{O}_{6}$, выращенных методом высокочастотного RF-напыления в атмосфере кислорода на подложке $\mathrm{Al}_{2} \mathrm{O}_{3}$ (c-срез). Рентгеноструктурные исследования показали, что пленки $\mathrm{Ba}_{0.5} \mathrm{Sr}_{0.5} \mathrm{Nb}_{2} \mathrm{O}_{6}$ являются $c$-ориентированными, параметр $c$ элементарной ячейки составил 3.948(1) А. Эллипсометрическими измерениями подтверждено, что пленки SBN-50 характеризуются естественным направлением роста, которое совпадает с направлением оптической оси кристалла. Анализ результатов эллипсометрических измерений показал отсутствие переходного слоя на границе пленка-подложка; толщина нарушенного слоя на свободной поверхности пленки $7.5 \mathrm{~nm}$, коэффициент объемного заполнения оценивается как 0.625 .
\end{abstract}

DOI: $10.21883 /$ OS.2019.05.47654.167-18

\section{Введение}

Сегнетоэлектрические материалы длительное время успешно интегрируются в устройства функциональной электроники, медицинской ультразвуковой диагностики, дефектоскопии, СВЧ и пьезотехники. Однако стремительное развитие в последние десятилетия микро- и наноэлектроники привело к тому, что в физическом материаловедении большое внимание стало уделяться получению и исследованию свойств гетероструктур на базе тонких пленок сегнетоэлектриков (СЭ) [1]. Известно, что в крупнокристаллических СЭ (керамика, поли- и монокристаллы) изменение и оптимизация их свойств достигается преимущественно вариацией химического состава: изменением концентрации компонент в твердых растворах (ТР) или модифицированием [2]. В наноразмерных структурах на основе пленок СЭ это возможно путем изменения механизма роста пленки и/или толщины материала, создания подслоев $[1,3]$, что позволяет в рамках одной химической композиции не только варьировать свойства материала, но и формировать новые свойства, свойственные только тонким пленкам. К числу одних из наиболее перспективных в тонкопленочном состоянии СЭ-материалов, благодаря высоким значениям диэлектрических, пьезоэлектрических и оптических характеристик, относятся одноосные СЭ в виде $\mathrm{TP} \mathrm{Sr}_{1-x} \mathrm{Ba}_{x} \mathrm{Nb}_{2} \mathrm{O}_{6}\left(\mathrm{SBN}_{x}\right)$ [4]. Как показывает анализ литературы, оптические свойства ТP $\mathrm{SBN}_{x}$ достаточно мало изучены, при этом имеют место противоречия.
В частности, в $[5,6]$ отмечено снижение показателей преломления $n_{o}$ и $n_{e}$ в $\mathrm{SBN}_{x} / \mathrm{MgO}(x=0.25,0.60,0.75)$ в сравнении с монокристаллами близких составов [4], в то время как в [7] отмечено, что в гетероструктуpe $\mathrm{Ba}_{0.5} \mathrm{Sr}_{0.5} \mathrm{Nb}_{2} \mathrm{O}_{6} / \mathrm{Pt}(111) / \mathrm{Si}(001)$ материал пленки по оптическим характеристикам соответствует таковым в монокристалле $\left(\mathrm{Ba}_{0.5} \mathrm{Sr}_{0.5}\right) \mathrm{Nb}_{2} \mathrm{O}_{6}$.

Настоящая работа посвящена установлению закономерностей формирования структуры и оптических свойств пленок $\mathrm{Sr}_{0.50} \mathrm{Ba}_{0.50} \mathrm{Nb}_{2} \mathrm{O}_{6}(\mathrm{SBN}-50)$, выращенных на подложке $\mathrm{Al}_{2} \mathrm{O}_{3}$.

\section{Объекты, методы получения и исследования}

Газоразрядное RF-напыление пленок SBN на предварительно подготовленную под гетероэпитаксиальные осаждения подложку $\mathrm{Al}_{2} \mathrm{O}_{3}$ (c-plane, толщина $0.43 \mathrm{~mm}$, производитель „MONOCRYSTAL“, г. Ставрополь) производилось на установке для осаждения наноразмерных монокристаллических пленок сложных оксидов „Плазма 50СЭ“ по методике, описанной ранее в работах $[7,8]$. Керамическая мишень стехиометрического состава $\mathrm{Sr}_{0.50} \mathrm{Ba}_{0.50} \mathrm{Nb}_{2} \mathrm{O}_{6}$ была изготовленная в отделе ИМиНТ НИИФ ЮФУ.

Рентгендифракционный анализ проводили на дифрактометре „ДРОН-4-07“ м методом $\theta / 2 \theta$ ( $\mathrm{Cu} K_{\alpha}$-излучение). 
Эллипсометрические измерения выполнялись с помощью многоуглового отражательного эллипсометра на длине волны гелий-неонового лазера $\lambda=632.8 \mathrm{~nm}$. Спектры пропускания снимались в диапазоне длин волн 200-1000 nm при комнатной температуре спектрофотометром Shimadzu UV-50.

\section{Экспериментальные результаты и обсуждение}

На рентгенограммах $\theta-2 \theta$-сканирования (рис. 1) присутствовали только отражения от плоскостей (001) пленки $\mathrm{SBN}-50$ и (006) подложки $\mathrm{Al}_{2} \mathrm{O}_{3}$. Установлено, что пленка является поликристаллической, текстурированной, и в ней отсутствуют примесные фазы. Пленка формируется с преимущественной ориентацией в направлении кристаллографической оси [001], параллельной нормали к подложке (ось [006] подложки). По максимумам рефлексов (001) пленки определен параметр элементарной ячейки $c=0.3948 \mathrm{~nm}$. Полученный параметр несколько превышает параметр объемного SBN-50 $(c=0.3945 \mathrm{~nm})$.

Эллипсометрические измерения подтвердили, что исследуемые пленки характеризуются естественным направлением роста, которое совпадает с направлением оптической оси кристалла [001] (поворот плоскости падения зондирующего луча не приводит к изменению значений эллипсометрических углов $\psi$ и $\Delta$ ). Полученные по результатам измерений эллипсометрические углы и являются функциями оптических параметров пленки и подложки, толщины пленки и угла падения $\varphi$ эллиптически поляризованного света. Определение характеристик пленки при известных параметрах подложки сводится к решению основного уравнения эллипсометрии

$$
e^{i \Delta} \operatorname{tg} \psi=\frac{R^{(p)}}{R^{(s)}}
$$

где $R^{(p)}$ и $R^{(s)}$ - амплитудные коэффициенты отражения электромагнитной волны $p$ - и $s$-поляризаций.

В простейшем случае однослойной однородной и изотропной прозрачной пленки на прозрачной подложке

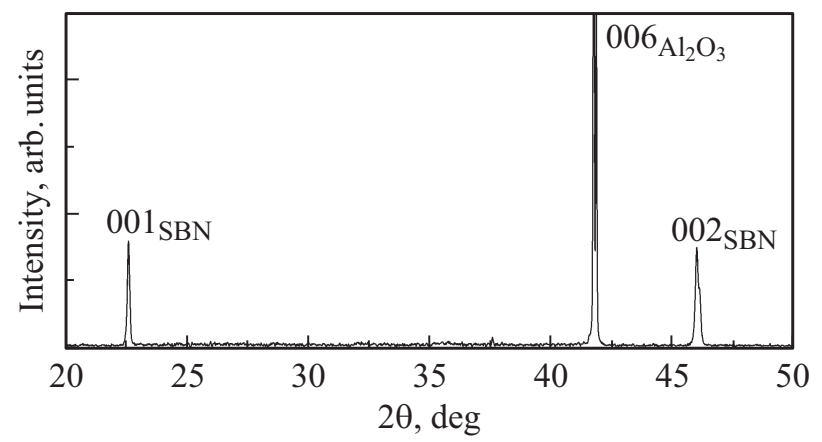

Рис. 1. $\theta-2 \theta$-рентгенограмма пленки $\mathrm{SBN} / \mathrm{Al}_{2} \mathrm{O}_{3}$.

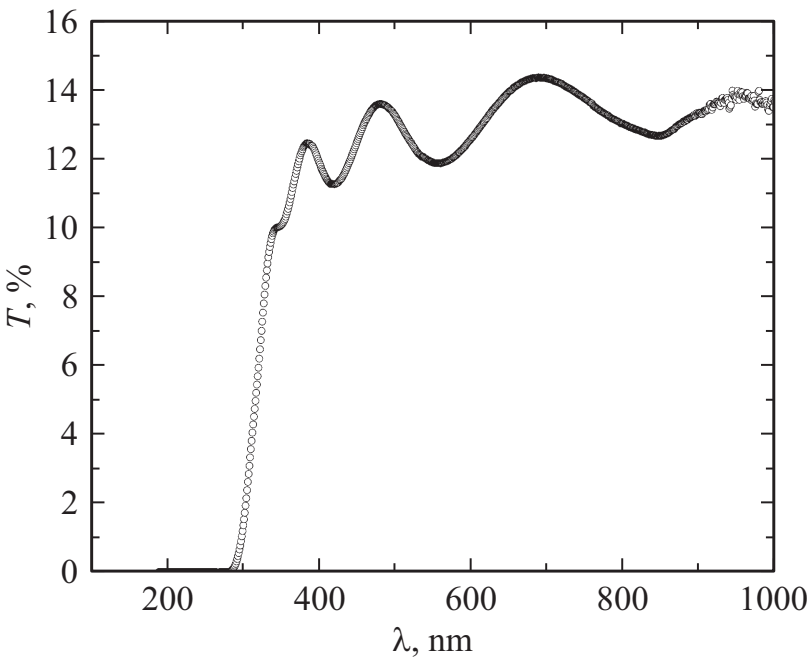

Рис. 2. Оптическое пропускание пленки $\mathrm{SBN}-50 / \mathrm{Al}_{2} \mathrm{O}_{3}$.

задача определения параметров пленки решается методом, изложенным в [9]. Метод основан на особенностях зависимостей $\psi(\varphi)$ и $\Delta(\varphi)$ : при угле падения, равном главному $\varphi=\varphi_{0}$, угол $\psi$ минимален, а $\Delta=\pi / 2$ или $3 \pi / 2$. При этом $\operatorname{Re}\left(\frac{R^{(p)}\left(\varphi_{0}\right)}{R^{(s)}\left(\varphi_{0}\right)}\right)=0$, и нахождение толщины пленки при известном показателе преломления сводится к решению квадратного уравнения относительно величины $\cos \alpha$, где аргумент $\alpha$ связан с фазой $\delta=\frac{4 \pi}{\lambda} d \sqrt{n^{2}-\sin ^{2} \varphi_{0}}$ соотношением $\alpha+2 \pi m=\delta$ $(m=0,1,2, \ldots-$ порядок интерференции). Для нахождения $m$ используются результаты, полученные измерениями спектра оптического пропускания.

Для интерпретации результатов эллипсометрических измерений пленки $\mathrm{SBN}-50 / \mathrm{Al}_{2} \mathrm{O}_{3}$ в нулевом приближении использовалась описанная методика. Эллипсометрические измерения дополнялись измерениями оптического пропускания в диапазоне длин волн 300-1000 nm (рис. 2). По анализу экстремумов восстановлен порядок интерференции $m=2$ при $\lambda=632.8 \mathrm{~nm}$. Процедура восстановления заключается в поиске такого минимального порядка интерференции для первого наблюдаемого длинноволнового экстремума, который обеспечивает дисперсию оптической толщины пленки $n_{0} d$, характерную для кристаллического материала. К особенностям описываемой SBN-пленки относится то, что для нее главный угол $\varphi_{0}=60.5^{\circ}$ и $\operatorname{tg} \varphi_{0}=1.73=n_{s} \quad\left(n_{s}-\right.$ показатель преломления подложки), т.е. зондирующий луч „не видит“ пленку. В этом случае толщина пленки такова, что френелевские амплитудные коэффициенты отражения воздух-пленка $r_{12}^{(p)}$, пленка-подложка $r_{23}^{(p)}$ и подложка-воздух $r_{31}^{(p)}$ удовлетворяют условию $r_{12}^{(p)}+r_{23}^{(p)}=r_{31}^{(p)}$, а $\cos \alpha=1$ и $d \sqrt{n^{2}-\sin ^{2} \varphi_{0}}=\lambda$. При этом в нулевом приближении для $n=n_{0}=2.3$ толщина пленки $d=297 \mathrm{~nm}$. 


\begin{tabular}{c|c|c|c|c|c|c|c}
\hline \multirow{2}{*}{$\begin{array}{c}\text { Подложка } \\
n_{s}\end{array}$} & \multirow{2}{*}{$\begin{array}{c}\text { Угол падения } \\
\varphi, \mathrm{deg}\end{array}$} & \multicolumn{2}{|c|}{ Эксперимент } & \multicolumn{2}{|c|}{ Изотропная модель } & \multicolumn{2}{|c}{ Анизотропная модель } \\
\cline { 3 - 8 } & $\psi, \mathrm{deg}$ & $\Delta, \mathrm{deg}$ & $\psi, \mathrm{deg}$ & $\Delta, \mathrm{deg}$ & $\psi, \mathrm{deg}$ & $\Delta, \mathrm{deg}$ \\
\hline \multirow{2}{*}{1.76} & 40 & 27.0125 & 182.7454 & 26.968 & 181.668 & 27.184 & 180.982 \\
& 45 & 21.3235 & 178.9224 & 21.22 & 179.997 & 21.575 & 179.155 \\
& 50 & 14.3796 & 173.4771 & 14.805 & 175.427 & 15.309 & 174.567 \\
& 55 & 8.3315 & 159.3511 & 8.205 & 161.605 & 8.817 & 161.635 \\
& 60 & 3.5288 & 100.6841 & 4.006 & 97.372 & 4.23 & 105.637
\end{tabular}

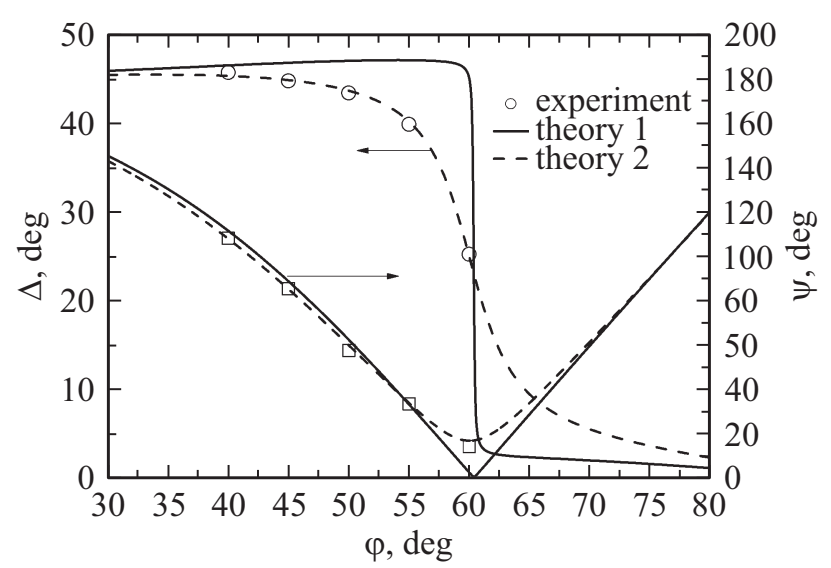

Рис. 3. Расчетные зависимости $\psi$ и $\Delta$ от угла падения $\varphi$ в модели однослойного покрытия с $n=2.3$ и $d=297 \mathrm{~nm}$ (theory 1) и экспериментальные значения для пленки $\mathrm{SBN} / \mathrm{Al}_{2} \mathrm{O}_{3}$; theory 2 - нарушенный слой с $d=7.5 \mathrm{~nm}, n_{e f}=1.7$, $k_{e f}<0.01$; пленка $-d=290 \mathrm{~nm}, n_{0}=2.3, n_{e}=2.28$.

Указанные особенности пленки позволяют достаточно просто оценить влияние анизотропии на интерпретацию результатов эллипсометрических измерений. Так как для необыкновенного луча фаза

$$
d^{(p)}=\frac{4 \pi}{\lambda} d \frac{n_{0}}{n_{e}} \sqrt{n_{e}^{2}-\sin ^{2} \varphi_{0}},
$$

то достаточно сравнить фазы $p$ - и $s$-составляющих отраженного света, т. е. сравнить величины $\sqrt{n_{e}^{2}-\sin ^{2} \varphi_{0}}$ и $\frac{n_{0}}{n_{e}} \sqrt{n_{e}^{2}-\sin ^{2} \varphi_{0}}$. Для монокристалла $\mathrm{Ba}_{0.5} \mathrm{Sr}_{0.5} \mathrm{Nb}_{2} \mathrm{O}_{6}$ $n_{0}=2.3-2.31 \quad$ и $\quad n_{e}=2.26-2.28 \quad[10,11]$. Тогда $\sqrt{n_{e}^{2}-\sin ^{2} \varphi_{0}}=2.129, \quad$ a $\quad \frac{n_{0}}{n_{e}} \sqrt{n_{e}^{2}-\sin ^{2} \varphi_{0}}=2.124$. Малое различие в значениях этих величин позволяет использовать изотропную модель SBN-пленки с $n m=n_{0}=2.3$ для интерпретации результатов эллипсометрических измерений.

Тот факт, что зондирующий луч „не видит“ пленку, приводит к тривиальным зависимостям $\psi(\varphi)$ и $\Delta(\varphi)$, рассчитанным в соответствии с основным уравнением эллипсометрии (1) в рамках оптически однородной изотропной пленки с $n=2.3$ и $d=297 \mathrm{~nm}$ на подложке с $n_{s}=1.73$ (рис. 3 , theory 1). Видно, что экспериментально найденные углы $\Delta(\varphi)$ значительно отклоняются от теоретической кривой 1 . Коррекция теоретической модели была осуществлена с учетом анизотропии пленки SBN-50, возможных переходных слоёв на границе пленка-подложка и на свободной поверхности пленки за счет ее шероховатости. При этом поверхностный переходный слой может обладать эффективным коэффициентом экстинкции $k_{f}$, отражающим потери энергии из-за ее рассеяния на неоднородностях поверхности пленки. Задача нахождения параметров переходных слоев решалась оптимизационным методом с несколькими параметрами минимизации по среднеквадратичным отклонениям [12]. Для границы пленка-подложка в качестве параметров поиска использовались толщина переходного слоя и его показатель преломления; для поверхностного нарушенного слоя, помимо его толщины и эффективного показателя преломления, учитывался и возможный эффективный коэффициент экстинкции. Расчеты показали отсутствие переходного слоя на границе пленка-подложка, что коррелирует с данными дифракции рентгеновских лучей. Неплохое соответствие между теорией и экспериментом в модели изотропной пленки было получено при введении поверхностного нарушенного слоя толщиной $n=7.5 \mathrm{~nm}$ с эффективным показателем преломления $n_{e f}=1.7$ и эффективным коэффициентом экстинкции $k_{e f}=0.01$. В модели эффективной среды Максвелла-Гарнета для прозрачной среды с показателем преломления $n_{e f}$ можно ввести

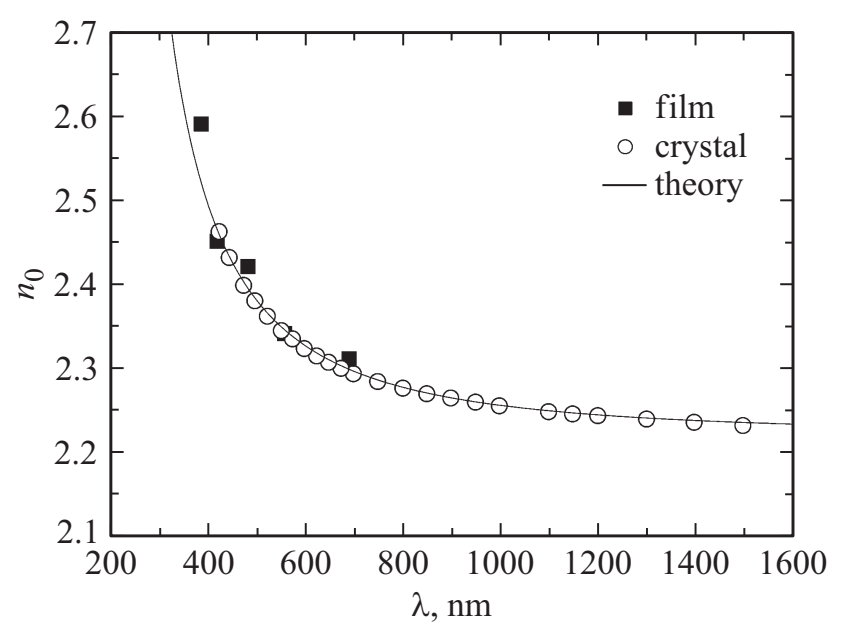

Рис. 4. Дисперсия показателя преломления пленки SBN$50 / \mathrm{Al}_{2} \mathrm{O}_{3}$ и монокристалла $\mathrm{SBN}-50[4]$. Сплошная линия расчеты по (5) с $\lambda_{0}=199 \mathrm{~nm}, S_{0}=0.99 \cdot 10^{14} \mathrm{~m}^{-2}$. 
коэффициент объемного заполнения $q$, определяемый соотношением [13]

$$
\frac{n_{e f}^{2}-1}{n_{e f}^{2}+2}=q \frac{n^{2} 0-1}{n^{2}+2} .
$$

Из (4) следует, что коэффициент объемного заполнения материалом пленки поверхностного нарушенного слоя составляет $q \approx 0.67$. Эффективному коэффициенту экстинкции 0.01 отвечают потери энергии вследствие ее рассеяния на неоднородностях поверхности менее $3 \%$.

Наилучшее согласие между экспериментом и теорией было получено при дополнительном учете анизотропии пленки с указанными выше параметрами нарушенного слоя и практически нулевым эффективным коэффициентом экстинкции (рис. 3, theory 2). В таблице представлены результаты эксперимента и рассчитанные с использованием полученных параметров поверхности зависимости $\psi(\varphi)$ и $\Delta(\varphi)$ для изотропной и анизотропной моделей пленки.

Определенная нами толщина пленки позволила найти из спектра оптического пропускания (рис. 2) дисперсию показателя преломления $n_{0}$ (рис. 4). Для аппроксимации зависимости в области слабого поглощения использовалось соотношение Зельмейера

$$
n_{0}^{2}(\lambda)-1=\frac{S_{0} \lambda_{0}^{2}}{1-\left(\lambda_{0} / \lambda\right)^{2}},
$$

где $\lambda_{0}-$ средняя длина волны, $S_{0}-$ средняя сила осциллятора.

Как видно из рис. 4, наблюдаемая в пленках SBN50 дисперсия практически совпадает с таковой для кристаллических материалов [4].

\section{Выводы и заключение}

1. В поликристаллических текстурированных пленках $\mathrm{SBN}-50 / \mathrm{Al}_{2} \mathrm{O}_{3}$, полученных одностадийным газоразрядным RF-напылением, методом дифракции рентгеновских лучей зарегистрировано небольшое увеличение параметpa $c$ элементарной ячейки по сравнению с объемным материалом. Установлено, что такая деформация элементарной ячейки не приводит к заметным отличиям в оптических свойствах пленок и монокристаллических объектов. Так, показатели преломления $n_{0}$ и $n_{e}$ на длине волны $\lambda=632.8 \mathrm{~nm}$, а также дисперсия $n_{0}(\lambda)$ пленки не отличаются от таковых в кристалле.

2. Результаты эллипсометрических измерений подтвердили, что направление роста пленки SBN-50 совпадает с направлением оптической оси кристалла. Анализ экспериментальных зависимостей эллипсометрических углов $\psi$ и $\Delta$ от угла падения показал, что пленка SBN-50 однородна, переходный слой на границе пленка-подложка отсутствует, на свободной поверхности имеется нарушенный слой. Наилучшее согласие между экспериментом и теорией достигается, если учитывать анизотропию пленки (ее толщина $290 \mathrm{~nm}$ ); при этом поверхностный нарушенный слой характеризуется толщиной $7.5 \mathrm{~nm}$ с коэффициентом объемного заполнения $q \approx 0.67$ и практически нулевыми потерями.

3. Полученные результаты можно распространить на тонкие пленки ниобатов бария стронция на подложках $\mathrm{Al}_{2} \mathrm{O}_{3}$, изготовленные описанным методом. Такие пленки целесообразно использовать в функциональных структурах на их основе.

\section{Финансирование работы}

Исследования выполнены в рамках реализации гос. задания ЮНЦ РАН (проект № 0120-1354-247), государственного задания Минобрнауки России (проект № 3.6371.2017/8.9) и при поддержке РФФИ (грант № 1629-14013).

\section{Список литературы}

[1] Воротилов К.А., Мухортов В.М., Сигов А.С. Интегрированные сегнетоэлектрические устройства. М.: Энергоатомиздат, 2011. $175 \mathrm{c}$.

[2] Фесенко Е.Г. Семейство перовскита и сегнетоэлектричество. М.: Атомиздат, 1972. 248 с.

[3] Мухортов В.М., Головко Ю.И., Бирюков С.В., Анохин А.С., Юзюк Ю.И. // ЖТФ. 2016. Т. 86. № 1. С. 93-98; Mukhortov V.M., Golovko Yu.I., Biryukov S.V., Anokhin A., Yuzyuk Yu.I. // Techn. Phys. 2016. V. 61. N 1. P. 91. doi $10.1134 / \mathrm{S} 106378421601014 \mathrm{X}$

[4] Кузьминов Ю.С. Сегнетоэлектрические кристаллы для управления лазерным излучением. М.: Наука, 1982. 400 с.

[5] Koo J., Kang E., Bae B. // J. Korean Physical Society. 2003. V. 42. P. S1161.

[6] Ковтун А.П., Зинченко С.П., Павленко А.В., Толмачев Г.Н. // Письма в ЖТФ. 2016. Т. 42. № 11. С. 48; Kovtun A.P., Zinchenko S.P., Pavlenko A.V., Tolmachev G.N. // Techn. Phys. Lett. 2016. V. 42. N 6. P. 577. doi 10.1134/S1063785016060109

[7] Толмачев Г.Н., Ковтун А.П., Захарченко И.Н., Алиев И.М., Павленко А.В., Резниченко Л.А., Вербенко И.А. // ФТТ. 2015. T. 57. № 10. C. 2050; Tolmachev G.N., Kovtun A.P., Zakharchenko I.N., Aliev I.M., Pavlenko A.V., Reznichenko L.A., Verbenko I.A. // Phys. Solid State. 2015. V. 57. N 10. P. 2106. doi 10.1134/S1063783415100339

[8] Павленко А.В., Захарченко И.Н., Анохин А.С., Куприна Ю.А., Киселева Л.И., Юзюк Ю.И. // ФТТ. 2017. T. 59. № 5. C. 888-891; Pavlenko A.V., Zakharchenko I.N., Anokhin A.S., Kuprina Yu.A., Kiseleva L.I., Yuzyuk Yu.I. // Phys. Solid State. 2017. V. 59. N 5. P. 909. doi 10.1134/S1063783417050249

[9] Грицких В.А., Жихарев И.В., Кара-Мурза С.В., Корчикова Н.В., Николаенко Ю.М., Тихий А.А. // Тр. 7-го Междунар. симп. „Физика поверхностных явлений, межфазных границ и фазовые переходы“. 2017. № 7. С. 53.

[10] Kip D., Aulkemeyer S., Buse K., Mersch F., Pankrath R., Krätzig E. // Phys. Stat. Sol. A. 1996. V. 154. N 2. P. K5. doi 10.1002/pssa.2211540235 
[11] Lenzo P.V., Spencer E.C., Ballman A.A. // Appl. Phys. Lett. 1967. V. 11. N 1. P. 23. doi 10.1063/1.1754944

[12] Тихий А.А., Грицких В.А., Кара-Мурза С.В., Корчикова Н.В., Николаенко Ю.М., Фарапонов В.В., Жихарев И.В. // Опт. и спектр. 2015. Т. 119. № 2. С. 282; Tikhii A.A., Gritskikh V.A., Kara-Murza S.V., Korchikova N.V., Nikolaenko Yu.M., Faraponov V.V., Zhikharev I.V. // Opt. Spectrosc. 2015. V. 119. N 2. P. 268. doi $10.1134 / \mathrm{S} 0030400 \mathrm{X} 15080238$

[13] Головань Л.А., Тимоченко В.Ю., Кашкаров П.К. // УФН. 2007. Т. 177. № 6. С. 619-638. doi 10.3367/UFNr.0177.200706b.0619; Golovan L.A., Timoshenko V.Yu., Kashkarov P.K. // Phys. Usp. 2007. V. 50. P. 595-612 doi 10.1070/PU2007v050n06ABEH006257 